\title{
Pronuclear score improves prediction of embryo implantation success in ICSI cycles
}

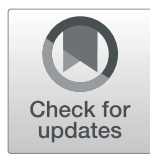

Sara Stigliani ${ }^{1 \dagger}$, Claudia Massarotti $^{2 \dagger}$, Francesca Bovis $^{3}$, Ida Casciano $^{1}$, Fausta Sozzi ${ }^{1}$, Valentino Remorgida ${ }^{2}$, Angelo Cagnacci ${ }^{2}$, Paola Anserini ${ }^{1}$ and Paola Scaruffi ${ }^{1 *}$

\begin{abstract}
Background: In assisted reproduction technology embryo competence is routinely evaluated on morphological criteria but efficacy remains relatively low. Additional information could be obtained by evaluating pronuclear (PN) morphology. Up to now controversial results have been reported about the prognostic value of PN score. One of the main limitations of literature data is the use of different PN classification methods. In this regard, in 2011 the ESHRE and Alpha Scientists in Reproductive Medicine defined three PN categories to standardize zygote assessment. In this study we evaluated whether the consensus ESHRE-Alpha system for the pronuclear scoring could be an useful additional criterion to improve prediction of embryo implantation potential.
\end{abstract}

Methods: This is a retrospective, longitudinal, observational, cohort study. We included 3004 zygotes from 555 women who underwent ICSI treatment at our Center between January 2014 and June 2019. The PN were categorized as score 1: symmetrical, 2: non-symmetrical, 3: abnormal. A subset of 110 zygotes did not cleaved. On day 2-3 1163 embryos were transferred, 232 arrested, and 9 were cryopreserved. Among the 1490 embryos cultured up to day 5-7, 516 became blastocysts: 123 were transferred on day 5 and 393 were cryopreserved. Comparisons of age, cleavage and blastocyst rate, quality of embryos, implantation success among PN score groups were evaluated by chi-square test or Kruskal-Wallis test as appropriate. Potential predictors of embryo implantation were first tested in univariable analysis using generalized estimating equations taking into account correlation between embryos originated from the same patient. Then, variables potentially associated with implantation success $(P<0.05)$ were included in a multivariable analysis for calculating the adjusted odds ratio (OR) and $95 \%$ confidence interval (CI).

Results: There was no significant difference in patients'age, cleavage and blastulation rates, and embryo morphology among the three PNscore groups. The PN score 1-embryos had a greater implantation success respect to score 2-3-ones ( $\mathrm{OR} 1.83 ; 95 \% \mathrm{Cl} 1.34-2.50, P=0.0001)$. Consistently, the pronuclear score remained predictive of implantation in top quality embryos (OR 1.68; 95\%Cl 1.17-2.42, $P=0.005$ ).

Conclusions: The consensus pronuclear score may be routinely included among criteria for embryo evaluation to increase patients' chance of becoming pregnant.

Keywords: Pronuclear morphology, Zygote, Blastocyst development, Implantation, Preimplantation embryo

\footnotetext{
* Correspondence: paola.scaruffi@hsanmartino.it

${ }^{+}$Sara Stigliani and Claudia Massarotti contributed equally to this work.

${ }^{1}$ UOS Physiopathology of Human Reproduction, IRCCS Ospedale Policlinico

San Martino, Largo R. Benzi, 10, 16132 Genova, Italy

Full list of author information is available at the end of the article
}

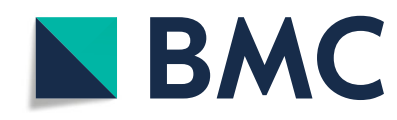

(c) The Author(s). 2021 Open Access This article is licensed under a Creative Commons Attribution 4.0 International License, which permits use, sharing, adaptation, distribution and reproduction in any medium or format, as long as you give appropriate credit to the original author(s) and the source, provide a link to the Creative Commons licence, and indicate if changes were made. The images or other third party material in this article are included in the article's Creative Commons licence, unless indicated otherwise in a credit line to the material. If material is not included in the article's Creative Commons licence and your intended use is not permitted by statutory regulation or exceeds the permitted use, you will need to obtain permission directly from the copyright holder. To view a copy of this licence, visit http://creativecommons.org/licenses/by/4.0/ The Creative Commons Public Domain Dedication waiver (http://creativecommons.org/publicdomain/zero/1.0/) applies to the data made available in this article, unless otherwise stated in a credit line to the data. 


\section{Introduction}

Identification of the embryo(s) with the highest implantation potential is a challenge not yet achieved in reproductive medicine, and it is a fundamental step for single embryo transfer approach. During the years, several approaches have been proposed for embryo viability evaluation, i.e. embryo morphokinetics, study of metabolic activity, prolonged culture, and both invasive and non-invasive preimplantation genetic testing [1-4]. Various embryological parameters are to some extent predictive of implantation potential. However, the overall success of these markers is still limited, with over $50 \%$ of transferred embryos failing to implant. Even invasive preimplantation genetic testing failed to improve overall pregnancy outcomes in a randomized controlled trial [5]. Thus, the search for new and reproducible markers of embryo viability is still in progress. For instance, it has been proposed that additional information on embryo viability potential could be obtained by evaluating pronuclear (PN) morphology based on zygote features 16-18 h after fertilization. Female pronucleus originates near the second polar body, whereas male pronucleus appears at the center of the cytoplasm. Following their formation, the female pronucleus migrates towards the male one until they are in close apposition. Nucleolar precursor bodies (NPB), randomly allocated within the pronuclei, appear shortly after fertilization and persist throughout the first cell cycles [6]. Unlike what was initially stated [7], the NPB are not precursors of nucleoli and they structurally support the formation of functional nucleoli when transcription starts in early embryos [6]. In the nucleoli, pre-ribosomal RNA (rRNA) synthesis occurs, the newly synthesized rRNAs are necessary for the translational process when the embryonic genome fully activates [8]. The progressive polarization of NPB controls the design of the embryonic axis, a fundamental step for cell determination in the developing embryo [9]. Alterations of these strictly related events may have abnormal consequences, including fertilization failure and uneven cleavage.

Starting from the first observations on PN, different classification systems have been proposed taking into account the PN size, the NPB position and alignment [10]. Although PN scores have been correlated with embryo development, pregnancy and implantation, to date there are conflicting evidences on the relationship between zygote morphology and IVF outcomes [10]. One of the main limitations of current literature is the use of different zygote grading systems. To this regard, in 2011 the ESHRE-Alpha consensus defined three PN categories to standardize the zygote assessment: symmetrical, nonsymmetrical and abnormal [1]. The symmetrical category includes zygotes showing two polar bodies, two centrally located and juxtaposed pronuclei, equal size and equivalent numbers and size of NPB equatorially aligned at the membrane juxtaposition. All the zygotes that do not have this ideal configuration belong to the non-symmetrical category. The abnormal category includes zygotes with no or one NPB. No studies verified the efficacy of such classification so far.

The aim of this retrospective study was to assess whether the consensus ESHRE system for the PN scoring could be a useful additional criterion to improve prediction of the embryo implantation potential.

\section{Methods \\ Study design, size, duration}

This is a retrospective, longitudinal, observational, cohort study. We included 3004 zygotes from 555 women (mean age: 35.6 years; range: 21-43) enrolled at our center between January 2014 and June 2019. The zygotes to be included in the study were selected on the basis of knowledge of their outcome: when 2 embryos from zygotes with different PN scores were transferred and only one implanted, these zygotes were excluded from the study. We included only homologous cycles using fresh eggs and ejaculated sperm. Another exclusion criterion was standard IVF, in order to standardize the fertilization check timing.

Embryo transfers (ET) were routinely performed on day 2-3 and, when available, two cleavage-stage embryos were transferred. Surplus embryos were cultured up to day 5-7 and those that developed up to blastocyst stage were cryopreserved. The ET were performed on day 5 in those cases with at least four good quality cleavage-stage embryos owing to the benefit from further observation in selecting the best embryos to transfer. On day 5 only elective single-embryo transfer were performed. In the study we included: (i) cleavage-stage ET; (ii) blastocyst-stage ET. Implantation success of each transferred embryo was defined as fetal cardiac activities at 12 weeks of gestation; miscarriages were excluded.

A STROBE (STrengthening the Reporting of OBservational studies in Epidemiology) checklist guideline is reported in Additional File 1.

\section{Outcomes measures}

The primary outcome was implantation success in relation to the PN score of (i) cleavage-stage embryos; (ii) blastocyst-stage embryos, and their predictive factors. The secondary outcomes were cleavage rate, quality of embryos, blastocyst development in relation to the PN score, and their predictive factors. We also evaluated the outcome of the newborns collecting their birthweights (expressed as percentile and SD-score for gestational age, according the World Health Organization reference curves). 


\section{Patients treatment}

Standard controlled ovarian stimulation protocols were used. Pituitary suppression was achieved with either Gonadotropin Releasing Hormone agonists or antagonists. Stimulation with gonadotropins was monitored by measuring serum estradiol levels and follicle growth. The trigger was either recombinant or urinary human chorionic gonadotropin or agonist trigger. Cumulus-oocyte complexes were collected $36 \mathrm{~h}$ later, washed in Sydney IVF Gamete buffer (Cook Medical, Sydney, Australia) and immediately incubated in Sydney IVF Fertilization medium (Cook Medical) at $37{ }^{\circ} \mathrm{C}$ in a humidified atmosphere of $6 \% \mathrm{CO}_{2}, 5 \% \mathrm{O}_{2}$ (Galaxy 48R incubators, New Brunswick Scientific, Edison, NJ, USA).

\section{Standard embryo culture}

After $2 \mathrm{~h}$ of incubation, the oocytes were denuded in HEPES-buffered medium (Sydney IVF Gamete medium, Cook Medical) containing $20 \mathrm{IU} / \mathrm{ml}$ of Hyaluronidase (Origio, Målov, Denmark). ICSI was performed immediately after denudation. Sperm samples were treated with a two-layer density gradient system (Sydney IVF Sperm Gradient, Cook Medical) or via Swim-up using Sydney IVF Gamete Buffer (Cook Medical). Incubations were performed at $37{ }^{\circ} \mathrm{C}$ in a humidified atmosphere of $6 \%$ $\mathrm{CO}_{2}, 5 \% \mathrm{O}_{2}$ (Galaxy 48R incubator; New Brunswick Scientific). Fertilization was assessed $16-18 \mathrm{~h}$ after injection (PN score 1: symmetrical, score 2: non-symmetrical, score 3: abnormal) [1], and embryos with two pronuclei were individually cultured from day 1 to day 3 into Sydney IVF Cleavage medium (Cook Medical) and from day 3 to day 5-7 in Sydney IVF Blastocyst medium (Cook Medical).

Standard day 2-3 embryo and blastocyst morphological assessment was carried out according to the current consensus system [1]. Arrested embryos were non-viable embryos in which development arrested for at least $24 \mathrm{~h}$, or in which all the cells degenerated or fragmented.

\section{Statistical analyses}

Descriptive statistics are reported as means \pm standard deviation (SD) for continuous variables and as absolute frequencies and percentages for categorical variables. Comparisons of age, cleavage rate, quality of embryos, blastocyst development, implantation success among PN score groups were evaluated by chi-square test or Kruskal-Wallis test as appropriate.

Potential predictors (PN score, cleavage-stage embryo morphology, patient's age, day of ET) of the study primary outcome (implantation versus non implantation) were first tested in univariable analysis using generalized estimating equations (GEE) with a logit link function for estimating the unadjusted odds ratio (OR) and $95 \%$ confidence interval $(95 \% \mathrm{CI})$ for the success of the implantation. Then, variables potentially associated with the success of the implantation in univariable comparisons $(P<0.05)$ were included in a multivariable analysis for calculating the adjusted OR for the success of the implantation. GEE models were used in order to take into account the correlation between embryos originated from the same patient and an unstructured correlation matrix was used as correlation structure.

Sensitivity analyses excluding PN score 3 embryos ( $n=21)$ or including only the top quality embryo $(n=860)$ were performed.

Analyses were carried out by MedCalc ${ }^{\ominus}$ software (Mariakerke, Belgium) and SAS 9.4 (SAS Institute, Cary, NC, USA). A $P$ value $<0.05$ was considered significant.

\section{Results}

Among the embryos originated from the 3004 zygotes enrolled in the study, 1163 embryos were transferred into uterus at their cleavage-stage, 1490 were cultured up to day 5-7, 232 arrested on day 3, 9 were cryopreserved on day 2-3; 110 zygotes did not cleave. Among the 1490 embryos whose culture was extended up to day 5-7, 516 became blastocysts: 123 were transferred into uterus on day 5 and 393 were cryopreserved (226 on day 5,156 on day 6 , and 11 on day 7 ).

\section{Relationship between PN score and embryo quality at cleavage stage}

A total of 2280 (76\%) score 1, 645 (21\%) score 2, and 79 (3\%) score 3 zygotes were obtained. There was not a significant difference in patients' age, cleavage rate and day 2-3 embryo morphology among the three PN score groups (Table 1). Moreover, the PN score did not always correlate with the embryo grade: only $60 \%$ top quality zygotes formed grade 1 embryos, and $54 \%$ poor quality zygotes (score 3) became high quality embryos (Fig. 1).

\section{Relationship between PN score and blastocyst development}

Blastocyst development rate was similar among the three PN score-derived embryo groups, without any statistically significant differences (Table 1). A total of $1106 \mathrm{PN}$ score 1-, 346 score 2-, and 38 score 3 -derived embryos were placed in extended culture. We did not find any reduction of blastulation rates in score $2-3(132 / 384$, $34 \%)$ groups respect to score 1 (384/1106, $35 \%)$ (Table 1). Notably, a single NPB was observed in the majority of the abnormal score $3 \mathrm{PN}$ category (75/79 cases) and $45 \%$ of cases (17/38) developed up to blastocyst stage when prolonged culture was performed.

First, we compared PN score and timing of blastulation. Among the 384 PN score 1-derived embryos that 
Table 1 Relationship between PN score and embryo quality parameters

\begin{tabular}{llll}
\hline PN score & $\mathbf{1}$ & $\mathbf{2}$ & $\mathbf{3}$ \\
\hline N. zygotes (\%) & $2280(76 \%)$ & $645(21 \%)$ & $79(3 \%)$ \\
Patients' age (mean \pm SD, years) & $35.7 \pm 4.4$ & $35.3 \pm 4.8$ & $34.4 \pm 4.1$ \\
Cleavage rate & $97 \%$ & $96 \%$ & $94 \%$ \\
N. grade 1 embryos (\%) & $1325 / 2280(58 \%)$ & $320 / 645(52 \%)$ & $42 / 79(53 \%)$ \\
N. grade $\mathbf{2}$ embryos (\%) & $578 / 2280(25 \%)$ & $173 / 645(27 \%)$ & $18 / 79(23 \%)$ \\
N. grade 3 embryos (\%) & $139 / 2280(6 \%)$ & $70 / 645(11 \%)$ & $3 / 79(4 \%)$ \\
N. arrested cleavage-stage embryos (\%) & $161 / 2280(6 \%)$ & $55 / 645(8 \%)$ & $11 / 79(14 \%)$ \\
Blastulation rate & $384 / 1106(35 \%)$ & $114 / 346(33 \%)$ & $18 / 38(47 \%)$ \\
\hline
\end{tabular}

reached the blastocyst stage, 191 (50\%) showed blastocoele expansion (grade $\geq 3$ ) on day 5; 77 (20\%) embryos were early blastocysts on day 5, and 116 (30\%) full blastocysts later on day 6. Similarly, among the $132 \mathrm{PN}$ score 2- and 3-derived embryos that reached the blastocyst stage, 59 (45\%) showed blastocoele expansion on day $5,25(19 \%)$ were early blastocysts on day $5(n=22)$ or day $6(n=3)$, and $48(36 \%)$ full blastocysts later on day 6 (Fig. 2a).

Second, we stratified embryos according to ICM and TE morphological grade. From PN score 1-derived embryos there were 232/384 (60\%) ICM grade A blastocysts (153 on day 5,79 on day 6-7), 68/384 (18\%) ICM grade B blastocysts ( 35 on day 5,33 on day $6-7$ ), and $7 /$ 384 (2\%) ICM grade C blastocysts (3 on day 5, 4 on day 6). Similarly, from $\mathrm{PN}$ score 2 and 3-derived embryos there were $77 / 132$ (58\%) ICM grade A blastocysts (42 on day 5,35 on day $6-7), 26 / 132$ (20\%) ICM grade B blastocysts (15 on day 5,11 on day $6-7$ ), and $4 / 132$
(3\%) ICM grade C blastocysts (2 on day 5 and 2 on day 6-7) (Fig. 2b).

From PN score 1-derived embryos there were 127/384 (33\%) TE grade A blastocysts (89 on day 5, 38 on day 6-7), 154/384 (40\%) TE grade B blastocysts (94 on day 5 , 60 on day $6-7$ ), and 26/384 (7\%) TE grade C blastocysts ( 8 on day 5,18 on day $6-7$ ). From PN score 2 - and 3 -derived embryos there were 51/132 (39\%) TE grade A blastocysts (32 on day 5, 19 on day 6), 49/132 (37\%) TE grade B blastocysts (24 on day 5,25 on day $6-7$ ), and $7 /$ 132 (5\%) TE grade $C$ blastocysts (3 on day 5, 4 on day 6-7) (Fig. 2c).

\section{Association between PN score and implantation success} We analyzed 1150 transferred embryos from 476 patients, in which the outcome for all embryos was known. Due to the paucity of score $3 \mathrm{PN}$ zygotes, we merged score $2(n=197)$ and score $3(n=21)$ PN-derived embryos in the subsequent analyses.

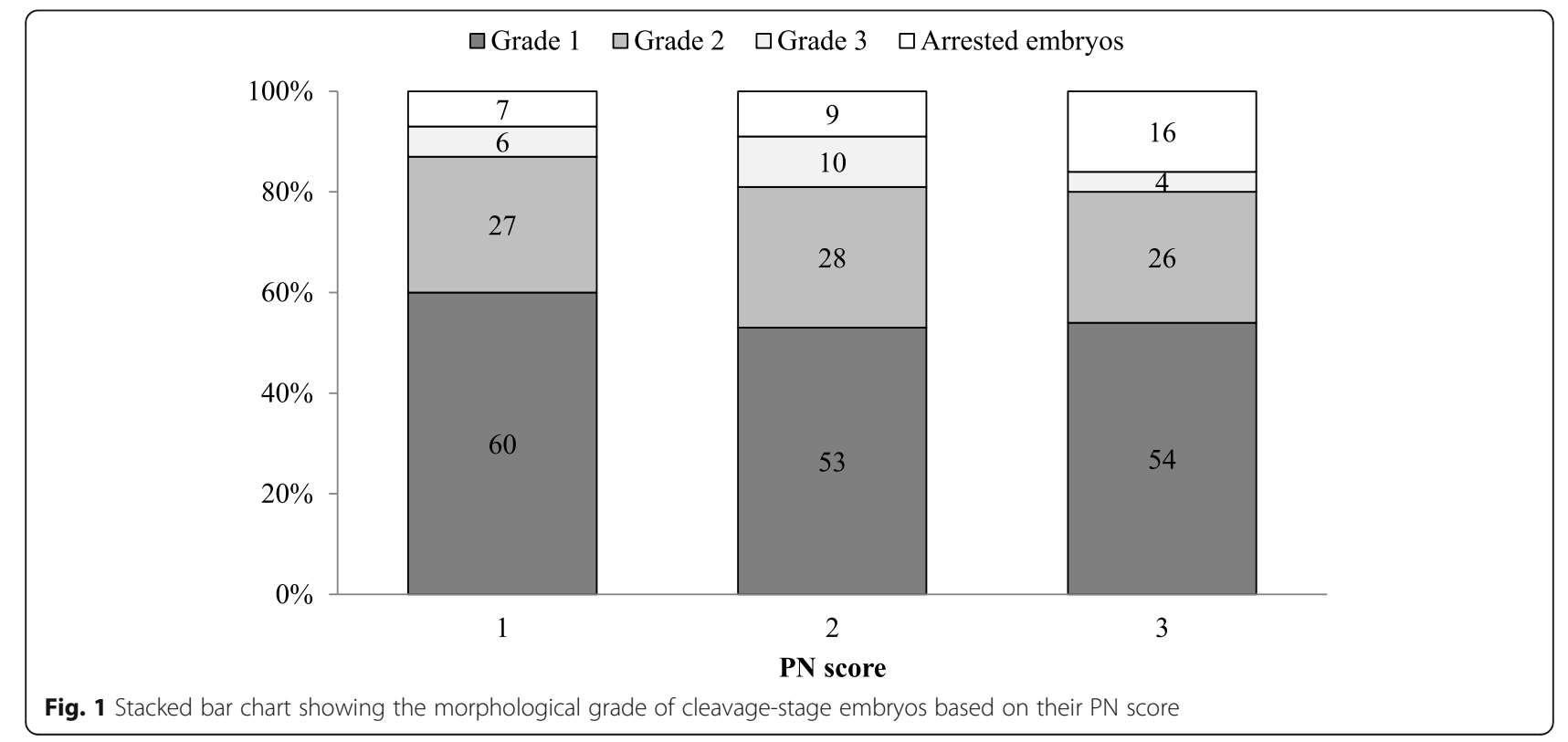


$\square$ PN score $1 \square$ PN score 2-3

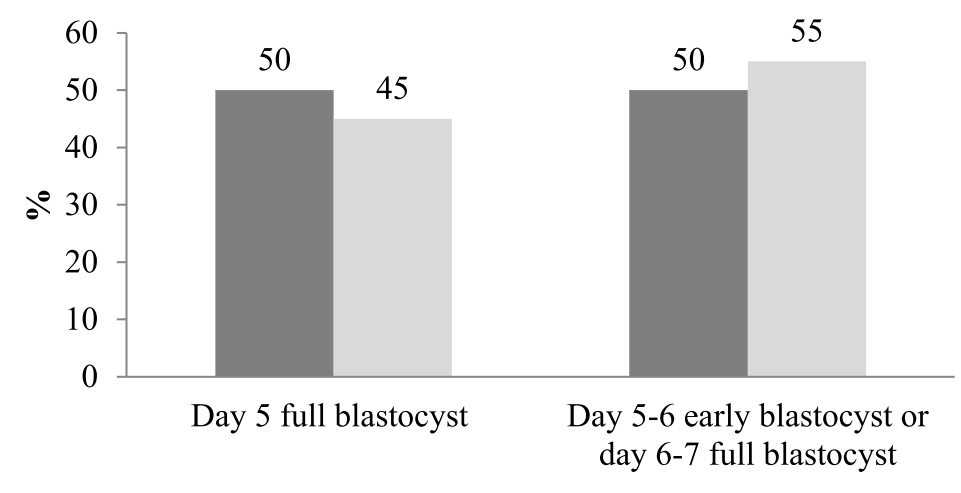

$\square$ PN score $1 \square$ PN score 2-3
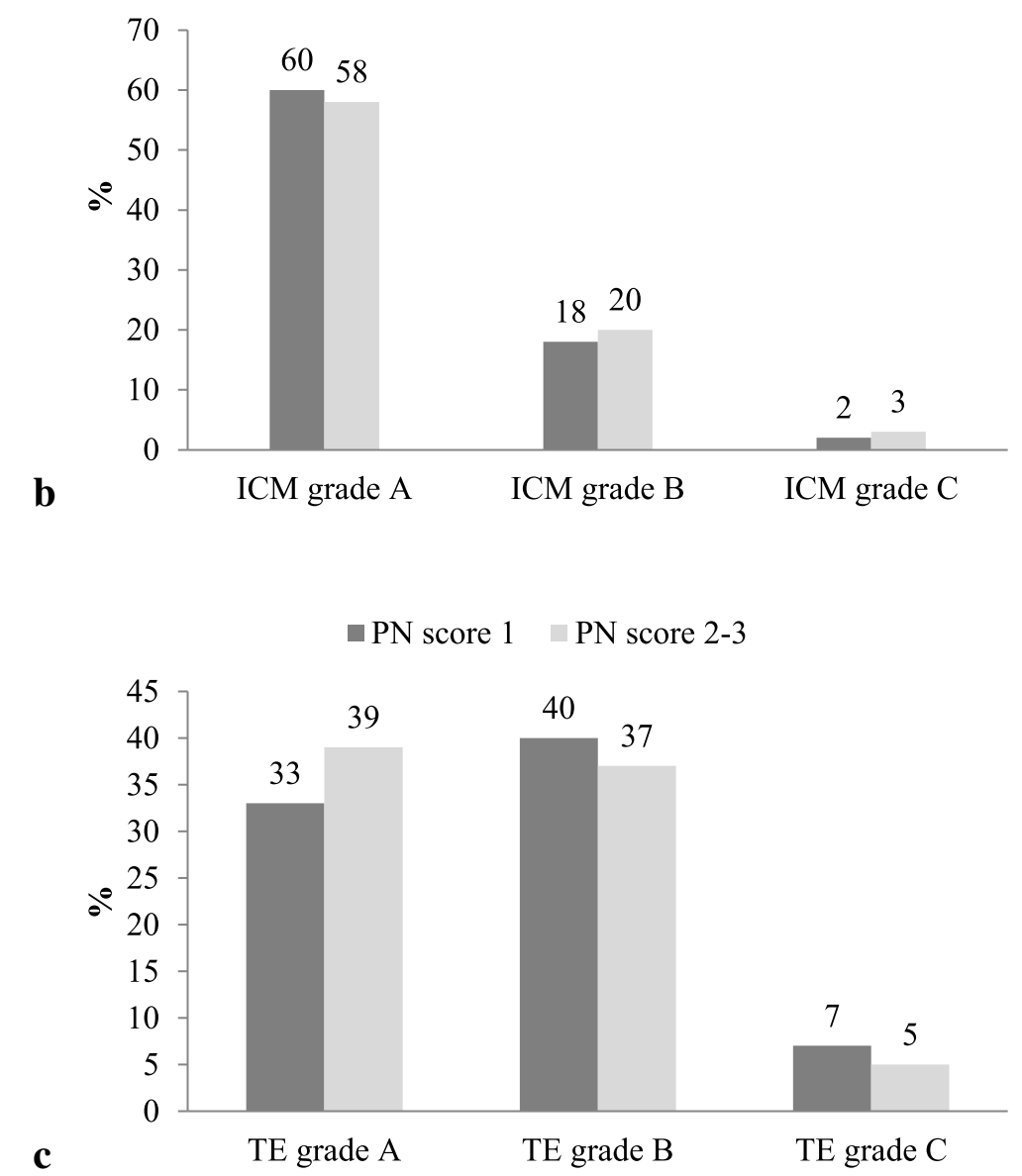

Fig. 2 Bar graphs comparing blastocysts parameters based on their PN score. Legend: Panel a: timing of blastulation; Panel b: ICM morphological grade; Panel c: TE morphological grade

A greater implantation success was observed for PN score 1- respect to PN score 2-3-derived embryos (15 and 9\%, respectively, $P=0.0121)$. This behavior was observed for both ET types, with statistically significant differences reached for the cleavage-stage group ( $15 \%$ vs. $7 \%, P=0.0043)$, due to its numerousness (Fig. 3). Three out of 21 transferred abnormal score 3 PN zygotes - specifically with a single NPB - were observed in the implanted embryo group.

Factors associated to the implantation at univariable and multivariable analysis are reported in Table 2. A PN 
$\square$ PN score $1 \square$ PN score 2-3

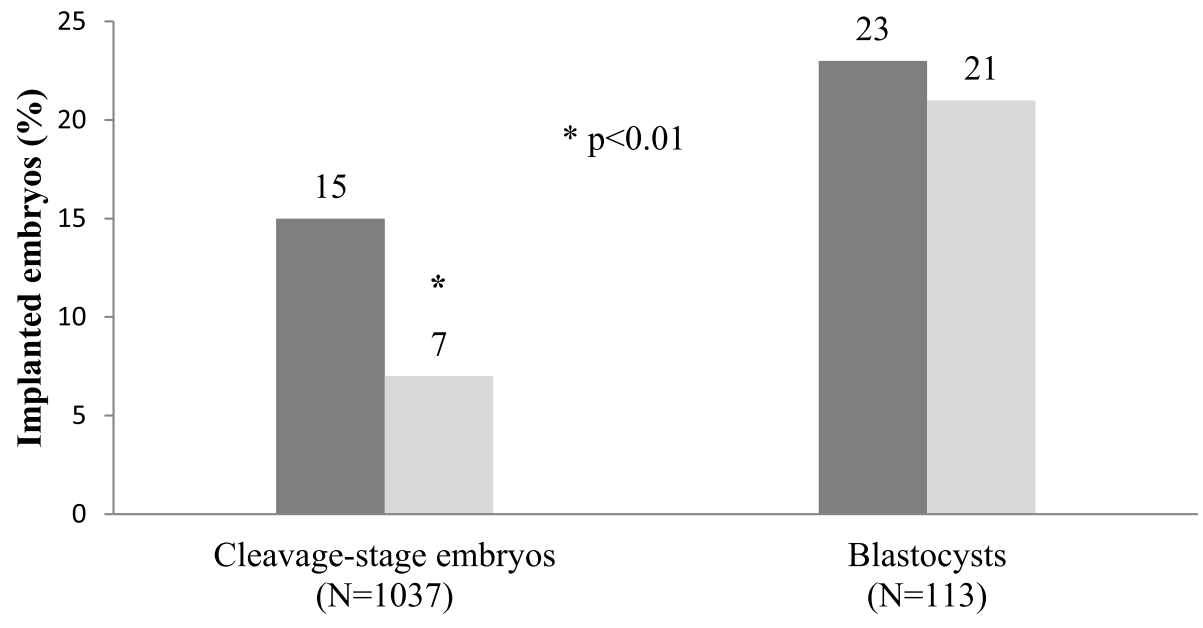

Fig. 3 Bar graph comparing the percentage of embryo implanted accordingly to their PN score

score $1 \quad(\mathrm{OR}=1.76 ; 95 \% \mathrm{CI}: 1.31-2.37, \quad P=0.0002)$, a cleavage stage embryo morphology equal to 1 (OR $=$ 4.78; $95 \% \mathrm{CI}: 1.84-12.42, P=0.001)$, age less than 35 years $(\mathrm{OR}=2.85 ; 95 \% \mathrm{CI}: 1.91-4.24, P<0.0001)$ and ET on day $5(\mathrm{OR}=2.12$; $95 \% \mathrm{CI}: 1.32-3.39, P=0.002)$ were factors associated to a successful implantation. A cleavage stage morphology equal to 2 showed a trend for the success of the implantation $(\mathrm{OR}=2.43$; $95 \% \mathrm{CI}$ : $0.90-$ $6.55, P=0.08)$. The multivariable analysis confirmed the findings of the unadjusted associations.

A sensitivity analysis excluding PN score 3 embryos $(n=$ 21) was also performed and no differences in unadjusted and adjusted ORs were found (see Additional file 2).

In a sensitivity analysis including only the top quality embryos $(n=860)$ we did not find any differences in unadjusted and adjusted ORs (Table 3).

\section{Perinatal characteristics of newborns}

We excluded from this analysis the outcome of ET of two embryos with discordant PN scores that resulted in delivery of only one baby. A total of 104 neonatal outcomes from PN score 1 embryos (of which 15 from twin gestations) and 4 from PN score 2 embryos (all from single pregnancies) were available. As detailed in Additional file 3 no statistical analyses could be performed due to the small number of evaluable newborns in PN score 2 group. We observed absence of twin pregnancies in PN score 2 category: this accounts for the higher birthweights and longer gestational period in PN score 2 respect to PN score1 group. In PN score 1 group, 8 pregnancies started as dichorionic-diamniotic twin pregnancies, but resulted in the live birth of only one of the fetuses. In fact, in 7 cases there was a spontaneous first trimester abortion of one twin, and in one case the patient opted for an elective pregnancy termination for a congenital mega bladder. Among singleton pregnancies, there was another elective termination for a major central nervous system malformation. No stillbirths as well as no malformations were recorded among the newborns of both score groups.

Table 2 Univariable and multivariable analysis of predictors of implantation success for all embryos $(n=1150)$

\begin{tabular}{|c|c|c|c|c|c|}
\hline & & Unadjusted OR (95\% Cl) & $p$-value & Adjusted OR (95\% Cl) & $p$-value \\
\hline \multirow[t]{2}{*}{ PN score } & $2-3$ (ref) & - & & & \\
\hline & 1 & $1.76(1.31-2.37)$ & 0.0002 & $1.83(1.34-2.50)$ & 0.0001 \\
\hline \multirow[t]{3}{*}{ Cleavage stage morphology } & 3 (ref) & - & & & \\
\hline & 1 & $4.78(1.84-12.42)$ & 0.001 & $4.20(1.68-10.48)$ & 0.002 \\
\hline & 2 & $2.43(0.90-6.55)$ & 0.080 & $2.16(0.84-5.59)$ & 0.111 \\
\hline \multirow[t]{2}{*}{ Age } & $\geq 35$ (ref) & - & & & \\
\hline & $<35$ & $2.85(1.91-4.24)$ & $<0.0001$ & $2.87(1.93-4.27)$ & $<0.0001$ \\
\hline \multirow[t]{2}{*}{ ET day } & Day 2-3 (ref) & - & & & \\
\hline & Day 5 & $2.12(1.32-3.39)$ & 0.002 & $1.79(1.09-2.93)$ & 0.020 \\
\hline
\end{tabular}


Table 3 Univariable and multivariable analysis of predictors of implantation success for top quality embryos $(n=860)$

\begin{tabular}{|c|c|c|c|c|c|}
\hline & & $\begin{array}{l}\text { Unadjusted OR } \\
(95 \% \mathrm{Cl})\end{array}$ & $p$-value & $\begin{array}{l}\text { Adjusted OR } \\
(95 \% \mathrm{CI})\end{array}$ & $p$-value \\
\hline \multirow[t]{2}{*}{ PN score } & 2-3 (ref) & - & & & \\
\hline & 1 & $1.60(1.12-2.30)$ & 0.011 & $1.68(1.17-2.42)$ & 0.005 \\
\hline \multirow[t]{2}{*}{ Age } & $\geq 35$ (ref) & - & & & \\
\hline & $<35$ & $2.81(1.86-4.25)$ & $<0.0001$ & $2.80(1.85-4.23)$ & $<0.0001$ \\
\hline \multirow[t]{2}{*}{ ET day } & Day 2-3 (ref) & - & & & \\
\hline & Day 5 & $1.88(1.16-3.04)$ & 0.010 & $1.71(1.03-2.84)$ & 0.039 \\
\hline
\end{tabular}

\section{Discussion}

To date, literature data about the correlation among zygote morphology, biological and clinical outcomes are inconclusive, mainly due to different methods used for PN scoring, time of PN observation, and insemination procedure [10].

This is the first study that evaluated the prognostic effect of the ESHRE consensus PN scoring system by a multivariable analysis. We did not find any relationship among PN score, embryo quality at cleavage and blastocyst stages, and blastulation rate, in line with some previous studies [11-17]. Intriguingly, half embryos from a single NPB zygote developed up to blastocyst stage and three successfully implanted. These data suggest that embryos derived from abnormal PN zygotes have some development potential, as described in literature using time-lapse imaging [18].

We demonstrated that the PN score improves prediction of implantation of cleavage-stage good morphology embryos. Moreover, PN scores 2 and 3 were associated with a lower implantation success, even though the morphology of the embryos was good. Based on these findings we argue that PN score may provide a noninvasive, early criterion helpful for selecting the best embryo(s) for transfer, particularly when multiple embryos of similar quality are available.

Overall, the multivariable analysis including potential confounding factors associated with the occurrence of pregnancy found that the PN score 1- along with top quality cleavage stage embryo morphology, patient's age less than 35 years and ET on day 5 - was predictive of embryos' implantation success.

The 2 and 3 PN patterns may be characterized by asynchrony in the formation and polarization of pronuclei. Such alteration of sequential, linked events can be at the origin of chromosomal abnormalities, whose consequences may appear at the implantation phase, after the embryonic genome activation [19-21]. This proposition would explain why we found a significant positive association between PN score 1 and implantation, without any evident effect of PN morphology on in vitro embryo developmental potential.
At pronuclear stage check, most embryos are at $\mathrm{S}$ or G2 phase when chromosomes are interconnected via nucleoli. As it has been already reported by some authors [22-24], if zygotes have different PN size and non-synchronous NPB there is an increased risk of embryo aneuploidy, due to aberrant chromosome duplication and division. Therefore, in absence of a genetic embryo assessment the PN score may help selection of those top quality embryos that have a chromosomal euploid set.

As observed in this study, top quality zygotes can become low quality embryos as well as top quality embryos can develop from low quality zygotes. In other words, neither zygote, nor embryo morphology alone are fully predictive of IVF outcome. Therefore, we believe that a combination of assessments and scores, including the PN score, may be helpful in non-invasive embryo selection.

Time-lapse monitoring of embryo development showed that PN morphology changes within a short time, at 16$20 \mathrm{~h}$ after ICSI, mostly from an asymmetry of NPB towards a symmetric or perfectly aligned distribution [18]. Therefore, a single microscopy observation may be misleading and such changes may partially explain the contradictory literature, where some authors reported no benefit when static observations of PN were performed [14, 25, 26]. Despite the dynamicity of the PN formation, our findings could be nonetheless useful for the majority of laboratories without of time-lapse technology availability. Certainly, the lack of standardization in the observation timing remains a critical issue for $\mathrm{PN}$ scoring, including the variability due to the method used for insemination. In fact, pronucleus development has an average delay of $4 \mathrm{~h}$ after conventional IVF as compared to ICSI, likely because the spermatozoon needs time to pass through the cumulus and corona cells and the zona pellucida [27]. This is the reason why we have excluded standard IVF cycles. We believe that the retrospective nature of this study could hardly have affected the reliability of our findings since at our laboratory all procedures were done by the same embryologists, who did not change throughout the duration of the study and applied the same protocols, including consistent timing of PN assessment. 


\section{Conclusions}

This is the first study of correlation between PN morphology and embryo implantation success, applying the PN score system proposed by ESHRE and a multivariable analysis which evaluated various potential confounding factors. Although validation through randomized perspective studies is needed, our findings suggest that the PN score could represent the earliest point at which the quality of the fertilized oocyte can be non-invasively evaluated and that the PN score may be routinely included among criteria for embryo evaluation. In this way, an evaluation based on the combination of both zygote and cleavage-stage morphology could assist in selecting the top quality embryo(s) with the highest chances of implantation. This could be of great value for all laboratories performing clinical IVF without any preimplantation genetic testing means.

\section{Abbreviations}

HEPES: 4-(2-hydroxyethyl)-1-piperazineethanesulfonic acid; ET: Embryo transfers; ESHRE: European Society of Human Reproduction and Embryology; ICM: Inner cell mass; ICSI: Intra cytoplasmic sperm injection; IVF: In vitro fertilization; NPB: Nucleolar precursor bodies; PN: Pronuclear; rRNA: Ribosomal RNA; SD: Standard deviation; TE: Trophectoderm

\section{Supplementary Information}

The online version contains supplementary material available at https://doi. org/10.1186/s12884-021-03820-7.

Additional file 1. STROBE reporting guideline of the study.

Additional file 2. Multivariable logistic analysis: predictors of implantationof PN score 1- vs. PN score 2-embryos ( $n=1084)$.

Additional file 3. Neonatal characteristics of newborns.

\section{Acknowledgements}

We are grateful to Mrs Laura Bacigalupo, Mr Martino Borra and Mrs Antonella Santi working as nurses at U.O.S. Physiopathology of Human Reproduction of Ospedale Policlinico San Martino, Genova.

\section{Authors' contributions}

SS collected biological data and contributed to critical discussion. CM collected clinical data and contributed to critical discussion. FB performed statistical analyses. IC performed ICSI cycles as embryologist. FS and PA performed patient recruitment and treatments. VR, PA, AC contributed to critical discussion. PS performed ICSI cycles as embryologist, designed the study, analyzed and interpreted the data, drafted the manuscript, and contributed to critical discussion. All authors read and approved the final article.

\section{Funding}

This research did not receive any specific grant from funding agencies in the public, commercial, or not-for-profit sectors.

\section{Availability of data and materials}

The datasets analyzed during the current study are available from the corresponding author upon request.

\section{Declarations}

\section{Ethics approval and consent to participate}

All procedures performed in studies involving human participants were in accordance with the ethical standards of the institutional and/or national research committee and with the 1964 Helsinki declaration and its later amendments or comparable ethical standards. The study was approved bythe Ethical Committee of Regione Liguria (approval n. 356/2019), and each couple signed a written informed consent.

Consent for publication

Not applicable.

\section{Competing interests}

The authors declare that they have no competing interests.

\section{Author details}

'UOS Physiopathology of Human Reproduction, IRCCS Ospedale Policlinico San Martino, Largo R. Benzi, 10, 16132 Genova, Italy. '2Department of Neurosciences, Rehabilitation, Ophthalmology, Genetics, Maternal and Child Health (DINOGMI), Academic Unit of Obstetrics and Gynecology, University of Genova, Genova, Italy. ${ }^{3}$ Department of Health Sciences (DISSAL), University of Genova, Genova, Italy.

Received: 2 October 2020 Accepted: 21 April 2021

Published online: 05 May 2021

\section{References}

1. Alpha Scientists in Reproductive Medicine and ESHRE Special Interest Group of Embryology. The Istanbul consensus workshop on embryo assessment: proceedings of an expert meeting. Hum Reprod. 2011;26:1270-1283.

2. Armstrong S, Bhide $P$, Jordan V, Pacey A, Farquhar C. Time-lapse systems for embryo incubation and assessment in assisted reproduction. Cochrane Database Syst Rev. 2018;5:CD011320.

3. Fragouli E, Wells D. Current status and future prospects of noninvasive preimplantation genetic testing for aneuploidy. Fertil Steril. 2018;110:408-9.

4. Viotti M, Victor AR, Zouves CG, Barnes FL. Is mitochondrial DNA quantitation in blastocyst trophectoderm cells predictive of developmental competence and outcome in clinical IVF? J Assist Reprod Genet. 2017;34:1581-5.

5. Munné S, Kaplan B, Frattarelli JL, Child T, Nakhuda G, Shamma FN, Silverberg K, Kalista T, Handyside AH, Katz-Jaffe M, Wells D, Gordon T, Stock-Myer S, Willman S. STAR Study Group. Preimplantation genetic testing for aneuploidy versus morphology as selection criteria for single frozen-thawed embryo transfer in good-prognosis patients: a multicenter randomized clinical trial. Fertil Steril. 2019;112:1071-9.e7.

6. Romanova L, Korobova F, Noniashvilli E, Dyban A, Zatsepina O. High resolution mapping of ribosomal DNA in early mouse embryos by fluorescence in situ hybridization. Biol Reprod. 2006;74:807-15.

7. Tesarik J, Kopecny V. Assembly of the nucleolar precursor bodies in human male pronuclei is correlated with an early RNA synthetic activity. Exp Cell Res. 1990;191:153-6.

8. Braude $\mathrm{P}$, Bolton $\mathrm{V}$, Moore $\mathrm{S}$. Human gene expression first occurs between the four and eight-cell stages of preimplantation development. Nature. 1988:332:459-61.

9. Edwards RG, Beard HK. Oocyte polarity and cell determination in early mammalian embryos. Mol Hum Reprod. 1997;3:863-905.

10. Nicoli A, Palomba S, Capodanno F, Fini M, Falbo A, La Sala GB. Pronuclear morphology evaluation for fresh in vitro fertilization (IVF) and intracytoplasmic sperm injection (ICSI) cycles: a systematic review. J Ovarian Res. 2013;6:64

11. De Placido G, Wilding M, Strina I, Alviggi E, Alviggi C, Mollo A, Varicchio MT, Tolino A, Schiattarella C, Dale B. High outcome predictability after IVF using a combined score for zygote and embryo morphology and growth rate. Hum Reprod. 2002;17:2402-9.

12. Jaroudi K, Al-Hassan S, Sieck U, Al-Sufyan H, Al-Kabra M, Coskun S. Zygote transfer on day 1 versus cleavage stage embryo transfer on day 3: a prospective randomized trial. Hum Reprod. 2004;19:645-8.

13. Maille L, Bergere M, Lemoine E, Camier B, Prevost JF, Bourdrel JM, Hammoud I, Selva J, Vialard F. Pronuclear morphology differs between women more than 38 and women less than 30 years of age. Reprod Biomed Online. 2009;18:367-73.

14. Nicoli A Capodanno F, Moscato L, Rondini I, Villani MT, Tuzio A, La Sala GB. Analysis of pronuclear zygote configurations in 459 clinical pregnancies obtained with assisted reproductive technique procedures. Reprod Biol Endocrinol. 2010;8:77. 
15. Qian $\mathrm{YL}$, Ye YH, Xu CM, Jin F, Huang HF. Accuracy of a combined score of zygote and embryo morphology for selecting the best embryos for IVF. J Zhejiang Univ Sci B. 2008;9:649-55.

16. Salumets A, Hydén-Granskog C, Suikkari AM, Tiitinen A, Tuuri T. The predictive value of pronuclear morphology of zygotes in the assessment of human embryo quality. Hum Reprod. 2001;16:2177-81.

17. Tesarik J, Junca AM, Hazout A, Aubriot FX, Nathan C, Cohen-Bacrie P, Dumont-Hassan M. Embryos with high implantation potential after intracytoplasmic sperm injection can be recognized by a simple, noninvasive examination of pronuclear morphology. Hum Reprod. 2000; 15:1396-9.

18. Azzarello A, Hoest T, Mikkelsen AL. The impact of pronuclei morphology and dynamicity on live birth outcome after time-lapse culture. Hum Reprod. 2012;27:2649-57.

19. Alvarez C, Taronger R, García-Garrido C, de Merlo G. Zygote score and status 1 or 2 days after cleavage and assisted reproduction outcome. Int J Gynaeco Obstet. 2008;101:16-20.

20. Ebner T, Moser M, Sommergruber M, Tews G. Selection based on morphological assessment of oocytes and embryos at different stages of preimplantation development: a review. Hum Reprod. 2003;9:251-62.

21. Nagy ZP, Dozortsev D, Diamond M, Rienzi L, Ubaldi F, Abdelmassih R, Greco E. Pronuclear morphology evaluation with subsequent evaluation of embryo morphology significant increases implantation rates. Fertil Steril. 2003;80:67-74

22. Edirisinghe WR, Jemmott $R$, Smith C, Allan J. Association of pronuclear $Z$ score with rates of aneuploidy in in vitro-fertilised embryos. Reprod Fertil Dev. 2005;17:529-34.

23. Gámiz P, Rubio C, de los Santos MJ, Mercader A, Simón C, Remohí J, Pellicer A. The effect of pronuclear morphology on early development and chromosomal abnormalities in cleavage-stage embryos. Hum Reprod. 2003; 18:2413-9.

24. Gianaroli L, Magli MC, Ferraretti AP, Lappi M, Borghi E, Ermini B. Oocyte euploidy, pronuclear zygote morphology and embryo chromosomal complement. Hum Reprod. 2007;22:241-9.

25. Brezinova J, Oborna I, Svobodova M, Fingerova H. Evaluation of day one embryo quality and IVF outcome-a comparison of two scoring systems. Reprod Biol Endocrinol. 2009:3:7-9.

26. James AN, Hennessy S, Reggio B, Wiemer K, Larsen F, Cohen J. The limited importance of pronuclear scoring of human zygotes. Hum Reprod. 2006;21: 1599-604.

27. Nagy ZP, Janssenswillen C, Janssens R, De Vos A, Staessen C, Van de Velde $H$, Van Steirteghem AC. Timing of oocyte activation, pronucleus formation and cleavage in humans after intracytoplasmic sperm injection (ICSI) with testicular spermatozoa and after ICSI or in-vitro fertilization on sibling oocytes with ejaculated spermatozoa. Hum Reprod. 1988;13:1606-12.

\section{Publisher's Note}

Springer Nature remains neutral with regard to jurisdictional claims in published maps and institutional affiliations.

Ready to submit your research? Choose BMC and benefit from:

- fast, convenient online submission

- thorough peer review by experienced researchers in your field

- rapid publication on acceptance

- support for research data, including large and complex data types

- gold Open Access which fosters wider collaboration and increased citations

- maximum visibility for your research: over $100 \mathrm{M}$ website views per year

At $\mathrm{BMC}$, research is always in progress.

Learn more biomedcentral.com/submissions 